\title{
El desafío de la fibrosis quística en Paraguay: una visión del camino recorrido
}

\section{The challenge of cystic fibrosis in Paraguay: a look at the path traveled}

\author{
Lidia Garcete Mañotti ${ }^{1}$
}

La fibrosis quística (FQ) constituye un trastorno hereditario complejo multisistémico, de evolución crónica y de gran heterogenicidad clínica. Afecta a la mayoría de los órganos, siendo el páncreas y los pulmones los más comprometidos, determinando la morbimortalidad. Desde sus primeras descripciones se ha recorrido un largo camino, en el que, gracias a técnicas de biología y genética molecular, se ha identificado el defecto básico y se comienza a vislumbrar la posibilidad de curación. Ella refleja la evolución de la medicina de las últimas décadas, pues en poco más de 60 años se ha pasado de su delimitación clínica como nueva entidad nosológica, al conocimiento preciso de su etiología, su sustrato patológico y su fisiopatología. Paralelamente se ha producido una transformación en su historia natural y de constituir un proceso letal de la primera infancia, ha pasado a ser una enfermedad crónica, abierta a la esperanza de un tratamiento curativo. Con el conocimiento de su fisiopatología y del defecto básico se desarrollaron tratamientos más eficaces y la sobrevida media es hoy de más de 40 años en países desarrollados ${ }^{(1,2)}$.

Alguna vez considerada una enfermedad de la población caucásica europea, es actualmente reconocido que ningún grupo étnico se encuentra excluido. En Latinoamérica se han incrementado los casos y es previsible un aumento mayor con el screening y el reconocimiento de las formas moderadas $^{(3)}$. El descubrimiento del gen mutado de la proteína reguladora de la conductancia transmembrana de la FQ (CFTR siglas en inglés) en
1989 y la función de la proteína natural (wild- type) CFTR en 1992, esclareció el mecanismo fisiopatológico básico. Actualmente, existen más de 1900 mutaciones y cerca de $2 / 3$ de los casos corresponden a la mutación p.F508del ${ }^{(4)}$. La prevalencia difiere entre las poblaciones, así en Latinoamérica cada país posee un variado espectro ${ }^{(2)}$.

La incidencia depende del ambiente, la consanguineidad, las variaciones fenotípicas derivadas del tipo de mutación y del grupo étnico. Debido a la heterogeneidad étnica se observa amplia variación a nivel mundial. En Latinoamérica se reportan incidencias de 1/3.900, 1/6.573, 1/8.500 y $1 / 14.000$ nacidos $\operatorname{vivos}^{(3,5-7)}$. Gracias a una mayor capacitación, programas de screening neonatal, diagnóstico molecular y otros test diagnósticos se ha progresado en las tasas de diagnóstico y en el diagnóstico oportuno. A pesar de ello, el sub diagnóstico resta como desafío. Se precisa mayor uniformidad y acceso universal a herramientas de diagnóstico dentro y entre países ${ }^{(3,8)}$.

El screening neonatal ha permitido evaluar el impacto de la intervención temprana. El diagnóstico en las primeras semanas de vida posibilita la terapéutica específica oportuna, previene la desnutrición y reduce el daño pulmonar mejorando la salud a mayor edad. Los niños identificados con screening demostraron una clara ventaja en el Zscore talla/edad y Zscore de función pulmonar, resaltando la importancia de la detección neonatal ${ }^{(2)}$.

\footnotetext{
${ }^{1}$ Universidad Nacional de Asunción. Facultad de Ciencias Médicas. Asunción, Paraguay. 
A nivel mundial las Asociaciones de Lucha Contra la FQ han impulsado el desarrollo científico y tecnológico e implementado acciones a favor de esta condición incapacitante y onerosa, desde lo económico, físico y psíquico. En Paraguay el diagnóstico y tratamiento de la FQ ha sido realizado desde fines de la década de 1980 con grandes dificultades debido a la escasez de métodos diagnósticos a nivel del sistema público y al elevado costo del tratamiento, destacándose el sub diagnóstico y el abandono del tratamiento como los mayores problemas ${ }^{(1)}$. En la década de 1990 me cupo acompañar a un grupo de padres de niños con $F Q$, quienes abrumados por la situación de orfandad en que se encontraban, lideraron la creación de la Fundación Paraguaya de lucha contra la FQ (FUPAFI) que se constituye en el año 1995. En ese transcurso, diversas publicaciones en medios locales y en colaboración con colegas latinoamericanos se realizaron en el afán de dar a conocer nuestra realidad $^{(9-14)}$. Luego de un largo transitar, esfuerzo y perseverancia FUPAFI obtiene la personería jurídica por decreto n⿳017.518 del Poder Ejecutivo en el año $2002^{(15)}$

Los primeros reportes en el país reflejan el diagnóstico tardío, el estado nutricional deficiente y la mortalidad precoz ${ }^{(1)}$. En un estudio de casos de Garcete L los diagnósticos fueron tardíos, ninguno antes de los 10 meses de edad ${ }^{(16)}$. Otra investigación que incluyó 30 pacientes con $F Q$, del Servicio de Pediatría. Hospital de Clínicas. FCM. UNA, entre los años 1985 al 2000, encontró que cuanto más tardío el diagnóstico, mayor el deterioro nutricional y menor capacidad de recuperación. El reducido número de casos en esa serie, hacia presumir un subdiagnóstico ${ }^{(1)}$. En el año 2002 Garcete et al describen una media de edad al diagnóstico de 21 meses 25 sobre una población de 31 pacientes $^{(17)}$.

Es en el año 2003 que Paraguay da un salto en la historia de la FQ con la Ley 2138 y en el 2016 con la Ley 5732 del Programa Nacional de Detección Neonatal (PNDN) $)^{(18)}$ que establece la detección, diagnóstico y tratamiento obligatorio y gratuito de la FQ para recién nacidos ( $\mathrm{RN})$. $\mathrm{Al}$ inicio solo para RN con síntomas o antecedentes, desde el año 2015 se tamizan el $100 \%$ de las muestras del país y se provee tratamiento a pacientes ${ }^{(5)}$.
En el año 2016 sobre 84 pacientes registrados en el PNDN, se reporta una mediana de edad al diagnóstico de 4,9 meses, $64 \%$ diagnosticados antes de los 6 meses de vida, avance atribuible a la creación del PNDN. El 22\% diagnosticado por screening y el $78 \%$ por síntomas. Un porcentaje de estos $78 \%$ fueron derivados de otras instituciones ${ }^{(19)}$.

Escurra y col reportan en este número la incidencia de la FQ en Paraguay por tamizaje en RN y confirmación diagnóstica con test del sudor, procesadas en el PNDN en los años 2105-2017, arrojando incidencias de 1/ $6.591 \mathrm{RN}, 1 / 4.176 \mathrm{RN}$ y 1 /5.112 RN. Los resultados en esta serie no presentan diferencias significativas con las reportadas para la población hispánica de la región que oscilan entre $1 / 3000$ a $1 / 8500^{(5,6,8,16)}$.

A pesar de los avances, la FQ sigue caracterizada por déficit de crecimiento, daño pulmonar y muerte prematura $^{(2,20)}$. El pronóstico depende de la precocidad del diagnóstico y de una terapéutica oportuna y actualizada. Las piedras angulares del tratamiento son la nutrición óptima, la fisioterapia y el ejercicio, aunadas al tratamiento precoz de la afectación pulmonar y al nuevo arsenal dirigido al defecto básico ${ }^{(1)}$. El tratamiento de la disfunción de la CFTR constituye una realidad muy próxima o ya establecida en varios países ${ }^{(3)}$. Las intervenciones focalizadas sobre los defectos básicos de la CFTR y su inicio oportuno evitaría la acumulación de daño tisular mejorando la evolución en los afectados ${ }^{(2)}$. Una caracterización genética por regiones es esencial para maximizar las oportunidades ${ }^{(3)}$.

En nuestro medio se ha recorrido un largo camino brindando posibilidades diagnósticas para conocimiento de su incidencia y abordaje precoz, como también posibilidades terapéuticas. Aún hay progresos por realizar en lo que atañe al diagnóstico de los casos que han escapado al screening, y al manejo multidisciplinario de los pacientes, mejorando el conocimiento médico y estableciendo políticas claras que protejan la salud. Se requiere disponibilidad de métodos diagnósticos, estudios genéticos para mejor abordaje y asistencia a las familias ${ }^{(1)}$. El registro de pacientes es vital para monitorizar la incidencia y evolución de estos, facilitar la identificación de las necesidades 
particulares y orientar las acciones ${ }^{(3)}$. El derecho a la salud universal es una deuda que debemos saldar y

\section{REFERENCIAS BIBLIOGRÁFICAS}

1. Garcete L. El compromiso del aparato digestivo y las manifestaciones extradigestivas en la fibrosis quística: análisis clínico laboratorial. [Monografía]. Asunción: Facultad de Ciencias Médicas, Universidad Nacional de Asunción; 2002.

2. Van Devanter DR, Kahle JS, O'Sullivan AK, Sikirica S, Hodgkins PS. Cystic fibrosis in young children: A review of disease manifestation, progression, and response to early treatment. Journal of Cystic Fibrosis. 2016; 15(2):147-57.

3. Silva Filho LV, Castaños C, Ruíz HH. Cystic fibrosis in Latin America-Improving the awareness. J Cyst Fibros. 2016;15(6):791-793.

4. Puppo H, Von Oetinger A, Benz E, Torres-Castro R, Zagolín M, Boza ML, et al. Caracterización de la capacidad física en niños del Programa Nacional de FQ de Chile. Rev Chil Pediatr. 2018;89(5):638-643.

5. Ascurra M, Valenzuela A, SalinasM, RodríguezS, Porzio $\mathrm{G}$, Ortiz L, et.al. Incidencia de la fibrosis quística en Paraguay. 2019;46(1):6-10.

6. Ribeiro Silva LV, Castaños C, Ruíz HH. Cystic fibrosis in Latin America. Improving the awareness. Journal of Cystic Fibrosis. 2016; 15(6):791-793.

7. Perez MM, Luna MC, Pivetta O, Keyeaux G. CFTR gene analysis in Latin American CF patients: Heterogeneous origin and distribution of mutations across the continent. Journal of Cystic Fibrosis. 2007; 6(3):194-208.

8. Borrajo GJC. Pesquisa neonatal de enfermedades congénitas. [Tesis Doctoral]. Universidad Nacional de la Plata, La Plata: Facultad de Ciencias Exactas; 2011.

9. Macri C, Gentile AS, Manterola A. Estudio clínico epidemiológico Latinoamericano de la fibrosis quística (mucoviscidosis). Arch. argent. pediatr. 1992; 90(2): 111-118.

10. Macri C, de Gentile AS, Botelli M, Castorina C, D'Astolfo M, Di Yacobo M, et al. Cystic Fibrosis Latin sólo lo lograremos con el compromiso de todos.

American Multicentric Study. Am. Rev. Resp. Dis. 1993; 147 (Suppl):579.

11. Garcete L. Manejo nutricional del paciente con fibrosis quística. Resúmenes del VIII Congreso Latinoamericano y III Jornada Hispano latinoamericana de Fibrosis Quística. La Habana, Cuba; 1997;79-80.

12. Baruja D, Meza R, Zacur M, Mayor R, Agüero L. Fibrosis quística, forma de presentación poco frecuente. A propósito de un caso. Pediatr (Asunción). 2002; 29: (Suppl):25.

13. MSPyBS. Guía Clínica: Fibrosis Quística. Asunción, Paraguay: Programa de Detección Neonatal, Ministerio de Salud Pública y Bienestar Social; 2009.

14. Garcete L. Tratamiento de reposición enzimática (TRE) en FQ. En: Aspectos nutricionales y gastroenterológicos de la fibrosis quística. Documento final de Working Groups de LASPGHAN. 2013. p.35-43.

15. Paraguay. Decreto del Poder Ejecutivo № 17.518/2002. Declaración de personería jurídica de la Fundación Paraguaya de lucha contra la Fibrosis Quística (FUPAFI). 2002.

16. Garcete L. Fibrosis quística en la infancia. Pediatr (Asunción). 1987;15(1):29-34.

17. Garcete L, Corrales B. Evaluación nutricional de pacientes con FQ. Pediatr (Asunción). 2002; 29 (Suppl): 25.

18. Paraguay. Ley $N^{\circ}$ 5.732: De la Creación del Programa Nacional de Detección Neonatal y su ámbito de aplicación. 2016.

19. Ortiz L, Sanabria L, Ascurra M. Caracterización nutricional de niños y adolescentes con FQ. Pediatria (Paraguay). 2017; 44(3):205-217.

20. Cystic Fibrosis Foundation. Patient Registry: Annual Data Report: 2015. Bethesda, Maryland: Cystic Fibrosis Foundation; 2016. 